\title{
GLOBAL SOLUTIONS TO CROSS DIFFUSION PARABOLIC SYSTEMS ON 2D DOMAINS
}

\author{
DUNG LE AND VU THANH NGUYEN
}

(Communicated by Catherine Sulem)

\begin{abstract}
This paper studies global existence of cross diffusion systems on 2-dimensional domains. We assume a quadratic growth on the reaction part and show that a solution exists globally if and only if its total reaction energy does not blow up in finite time. Applications to cross diffusion systems with Lotka-Volterra reaction are presented.
\end{abstract}

\section{INTRODUCTION}

In this paper we consider the following cross diffusion system of $m$ equations:

$$
\begin{cases}u_{t}=\operatorname{div}(A(x) D u)+f(u) & \text { in } \Omega \times(0, T), \\ u=0 & \text { on } \partial \Omega \times[0, T], \\ u(x, 0)=U_{0}(x) & x \in \Omega .\end{cases}
$$

Here, $\Omega$ is a smooth and bounded domain in $\mathbb{R}^{2}$ and $T>0$. The solution $u=\left(u_{i}\right)_{i=1}^{m}$ is a vector valued function on $\Omega \times[0, T)$ and takes the given vector valued function $U_{0}$ as its initial value. We write $u_{t}, D u$ to denote respectively the temporal and spatial derivatives of $u$. The matrix $A(x)=\left(a_{i j}(x)\right)$ is a matrix $m \times m$ whose entries are smooth functions $a_{i j}$ on $\Omega$ and the reaction $f(u)$ is a vector valued function on $\mathbb{R}^{m}$.

Systems like (1.1) occur in many applications where the studied species are assumed to diffuse and interact with each other in the region $\Omega$. It happens that a great many physical (physico-chemical, biological, ecological, etc.) phenomena can be modeled by (1.1) where $u:=\left(u_{1}, \ldots, u_{m}\right)$ is the vector of densities (concentrations, population densities, temperatures, charged particle densities, etc.), $A(x) D u$ is the flux vector, and $f(u)=\left(f_{i}(u)\right)_{i=1}^{m}$ with $f_{i}(u)$ being the production/reaction rate for the $i$ th component $u_{i}$. For simplicity, in this paper we only discuss the zero Dirichlet boundary condition in (1.1) and the nonzero case can be reduced to this situation by a simple change of variables if the boundary data is sufficiently smooth. Furthermore, the Neumann or mixed boundary conditions can be treated as well with some minor changes and will be reported in a forthcoming paper [3], where we also discuss blow up solutions to (1.1).

Received by the editors November 12, 2013 and, in revised form, March 3, 2014.

2010 Mathematics Subject Classification. Primary 35J70, 35B65, 42B37.

Key words and phrases. Parabolic systems, global existence.

The first author was partially supported by NSF grant DMS0707229. 
In applications to biology and ecology phemomena, one of the most commonly assumed form of $f(u)$ is the Lotka-Volterra reaction which is defined by

$$
f_{i}(u)=u_{i}\left(b_{i}-\sum_{j=1}^{m} c_{i j} u_{j}\right), \quad u=\left(u_{i}\right)_{i=1}^{m} \quad \text { and } \quad i=1, \ldots, m
$$

The values of $b_{i}$ and $c_{i i}$ are the intrinsic birth or death rates of the $i$ th species. The value of $c_{i j}$, when $i \neq j$, represents the effect that the $j$ th species has upon the $i$ th species and the effect is proportional to the populations of both species.

When the matrix $A$ is diagonal, i.e. $a_{i j}(x) \equiv 0$ for $i \neq j$, (1.1) then reduces to the well-known Lotka-Volterra system of predator-prey models.

The local existence of classical solutions (1.1) with such reactions and full matrix $A$ was established by H. Amann in the seminal papers [1,2]. This result is summarized in the following theorem.

Theorem 1.1 ([1], 2]). Suppose $\Omega \subset \mathbb{R}^{n}, n \geq 2$, with $\partial \Omega$ being smooth. Let $p_{0} \in(n, \infty)$ and $u_{0}$ be in $W^{1, p_{0}} 1(\Omega)$. Then there exists a maximal time $T_{0} \in(0, \infty]$ such that the system (1.1) has a unique classical solution in $\left(0, T_{0}\right)$ with

$$
u \in C\left(\left[0, T_{0}\right), W^{1, p_{0}}(\Omega)\right) \cap C^{1,2}\left(\left(0, T_{0}\right) \times \bar{\Omega}\right) .
$$

Moreover, if $T_{0}<\infty$, then

$$
\lim _{t \rightarrow T_{0}^{-}}\|u(\cdot, t)\|_{W^{1, p_{0}(\Omega)}}=\infty
$$

Many efforts have been made to investigate the existence globally in time of solutions for (1.1). In some special cases with only two equations and very strong restrictions on the matrix $A$, the solutions are proved to exist globally in time (see [8,11). Despite these achievements, whether this full system possesses global time solutions or finite time blow up solutions remains challenging and vastly open, even for 2-dimensional domains. One of the key obstacles is that there is no maximum or comparison principles available for the system and proving that the $L^{\infty}$ norm of the solutions does not blow up is already a challenging problem.

In this paper, we will avoid the $L^{\infty}$ approach and show that if one can control the much weaker total reaction energy of the solution, then its BMO norm will not blow up. Thanks to the well-known fact that $B M O$ functions are in $L^{p}$ for all $p \in[1, \infty)$ we can apply the standard semigroup theory for (1.1) to show that (1.3) does not happen and global existence results then follow. Our main result stated in Section 2 is Theorem 1.1. We will also present its application to (1.1) with (1.2) in Theorem 2.3. Furthermore, as a cautionary note in using cross diffusion effects in modeling and justifying our assumptions, we will give an example of a sign changing solution for (1.1) in Section 4 which shows that comparison principles are generally unavailable for our systems.

Our paper is then organized as follows. We state our main structural conditions for (1.1) and theorems in Section 2, Section 3 is devoted to the technical lemmas as well as the proof of our theorems. We end the paper with the proof of the existence of sign changing solutions in Section 4 . 


\section{MAin RESUlts}

We state the main theorem of this paper under the following assumptions:

A): $A(x)$ is $C^{1+\theta}$ continuous for some $\theta>0$ and there is positive real $\lambda$ such that

$$
\lambda|\zeta|^{2} \leq\langle A(x) \zeta, \zeta\rangle \quad \forall x \in \Omega, \zeta \in \mathbb{R}^{m n} .
$$

F1): There is a constant $C_{1}$ such that

$$
\left|f_{u}(u)\right| \leq C_{1}(|u|+1) .
$$

Theorem 2.1. Suppose that $\mathbf{A})$ and $\mathbf{F 1 )}$ are satisfied. Let $u$ be a classical solution to (1.1). Suppose that there is a continuous function $\Phi(t)$ on $(0, \infty)$ such that

$$
\sup _{t \in(0, T)} \int_{0}^{t} \int_{\Omega}\langle f(u(x, s)), u(x, s)\rangle d x d s \leq \Phi(T) \quad \forall T \in\left(0, T_{0}\right),
$$

whenever $u$ exists on $\left(0, T_{0}\right)$.

Then $u$ exists globally on $(0, \infty)$.

We can refer to the integral of $\langle f(u), u\rangle$ over $\Omega \times(0, t)$ on the left of (2.2) as the total reaction energy of the solution $u$. The above theorem thus shows that if this energy does not blow up in finite time, then the solution $u$ exists globally. If $f(u)$ is of quadratic growth as in (1.2), then one can easily see that the condition (2.2) is equivalent to the boundedness of the kinetic energy of $u$,

$$
\sup _{t \in(0, T)} \int_{\Omega}|u|^{2} d x+\int_{0}^{T} \int_{\Omega}|D u(x, t)|^{2} d x d t,
$$

or that of

$$
\sup _{t \in(0, T)} \int_{\Omega}|u|^{2} d x .
$$

In fact, we will also show that

Corollary 2.2. Suppose that $\mathbf{A})$ and $\mathbf{F 1 )}$ are satisfied. Let u be a classical solution to (1.1). Suppose that there is a continuous function $\Phi(t)$ on $(0, \infty)$ such that there is $p>1$ such that

$$
\sup _{t \in(0, T)} \int_{\Omega}|u(x, t)|^{p} d x \leq \Phi(T) \quad \forall T \in\left(0, T_{0}\right),
$$

whenever $u$ exists on $\left(0, T_{0}\right)$. Then $u$ exists globally on $(0, \infty)$.

As an application of Theorem 1.1 we consider the following form of $f(u)=$ $\left(f_{i}(u)\right)_{i=1}^{m}$ :

$$
f_{i}(u)=u_{i}\left(b_{i}-\sum_{j=1}^{m} c_{i j}\left|u_{j}\right|\right), \quad u=\left(u_{i}\right)_{i=1}^{m} \quad \text { and } \quad i=1, \ldots, m .
$$

We will show that the total reaction energy of $u$ does not blow up and have the following result. 
Theorem 2.3. Assume A) and that $f(u)=\left(f_{i}(u)\right)_{i=1}^{m}$ is given by (2.4) for some positive constants $b_{i}, c_{i j}$. For any initial data $U_{0} \in W^{1, p}\left(\Omega, \mathbb{R}^{m}\right)$ with $p>2$ the following Cauchy problem:

$$
\begin{cases}u_{t}=\operatorname{div}(A(x) D u)+f(u) & \text { in } \Omega \times(0, \infty), \\ u=0 & \text { on } \partial \Omega \times[0, \infty), \\ u(x, 0)=U_{0}(x) & x \in \Omega\end{cases}
$$

has a classical solution that exists globally.

Concerning applications in biology and ecology, the components $u_{i}$ are usually population densities of the species under consideration. Therefore, it may be more favorable if one assumes positive initial data $U_{0}$ and drops the absolutes in (2.4) to conform to the standard Lotka-Volterra setting and consider

$$
f_{i}(u)=u_{i}\left(b_{i}-\sum_{j=1}^{m} c_{i j} u_{j}\right), \quad u=\left(u_{i}\right)_{i=1}^{m} \quad \text { and } \quad i=1, \ldots, m .
$$

Of course, Theorem 2.3 can immediately apply to this case if one can establish that $u$ stays nonnegative for all time, assuming also that the initial data are positive. This is a well-known result for diagonal systems but it is not the case for cross diffusion systems. Indeed, we will show that

Theorem 2.4. There are a full matrix $A$ and positive reals $b_{i}, c_{i j}$ and a positive initial data $U_{0}(x)$ such that the system (2.5) with $f(u)$ being given by (2.4) has a unique classical solution $u, v$ that exists globally and changes sign on $(0, \infty)$.

Obviously, this result shows that if one considers (2.6) in the system (2.5), then one may not expect global solutions that stay nonnegative for all time.

\section{TEChNiCAL LEMmas}

In this section, we discuss a priori estimates of solutions to (1.1) under our most general assumptions which will be verified later on.

We first recall the following Gagliardo-Nirenberg inequality for any bounded smooth domain $\Omega \subset \mathbb{R}^{n}(n \geq 2)$,

$$
\left(\int_{\Omega}|\phi|^{q} d x\right)^{\frac{1}{q}} \leq C(n)\left(\int_{\Omega}|\phi|^{r} d x\right)^{\frac{1-\alpha}{r}}\left(\int_{\Omega}|D \phi|^{2} d x\right)^{\frac{\alpha}{2}} \quad \forall \phi \in W^{1,2}(\Omega),
$$

and $q, r \geq 1$ are related by $\frac{1}{q}=\left(\frac{1}{2}-\frac{1}{n}\right) \alpha+\frac{1-\alpha}{r}$. When $n=r=2$, this relation becomes $\alpha=1-\frac{2}{q}$. If we take $q=4$, then $\alpha=\frac{1}{2}$ and the above can be rewritten as

$$
\int_{\Omega}|\phi|^{4} d x \leq C \int_{\Omega}|\phi|^{2} d x \int_{\Omega}|D \phi|^{2} d x \quad \forall \phi \in W_{0}^{1,2}(\Omega) .
$$

Let us start with the following lemma which shows that if a classical solution $u$ to (1.1) exists in some time interval its temporal derivative can be controlled by the rate of change of the reaction with respect to $u$.

Lemma 3.1. Let $u$ be a classical solution to (1.1) in some interval $\left(0, T_{0}\right)$. Assume that there is a continuous function $\Phi_{1}(t)$ on $(0, \infty)$ such that

$$
\int_{0}^{t} \int_{\Omega}\left|f_{u}(u(x, s))\right|^{2} d x d s \leq \Phi_{1}(t) \quad \forall t \in\left(0, T_{0}\right) .
$$


Then there is a continuous function $\bar{\Phi}(t)$ on $(0, \infty)$ depending on $\Phi_{1}$ such that

$$
\int_{\Omega}\left|u_{t}(x, t)\right|^{2} d x \leq \bar{\Phi}(t) \int_{\Omega}\left|u_{t}\left(x, t_{0}\right)\right|^{2} d x \quad \forall t_{0}>0, t \in\left(t_{0}, T_{0}\right) .
$$

In the proof and the rest of this paper we will use $C$ to denote a universal constant depending on the constant in (3.1) and (3.2). However, the value of this constant can change from line to line in our argument and does not effect the qualitative nature of our assertions. If needed, $C(\cdots)$ will be used to reflect the dependence of $C$ on essential parameters.

Proof. Because $u$ is smooth in the interior of $Q=\Omega \times\left(0, T_{0}\right)$, we can differentiate the system of $u$ with respect to $t$ and get

$$
u_{t t}=\operatorname{div}\left(A(x) D u_{t}\right)+f_{u}(u) u_{t} \quad \forall t \in\left(0, T_{0}\right) .
$$

Because $u=0$ on the lateral boundary of the cylinder $Q$, we see $u_{t}=0$ there too so that we can multiply $u_{t}$ to the above system and integrate the result in $x$ over $\Omega$ to obtain

$$
\int_{\Omega}\left\langle u_{t t}, u_{t}\right\rangle d x=\int_{\Omega}\left\langle\operatorname{div}\left(A(x) D u_{t}\right), u_{t}\right\rangle d x+\int_{\Omega}\left\langle f_{u}(u) u_{t}, u_{t}\right\rangle d x, \quad \forall t \in\left(0, T_{0}\right) .
$$

Since $u_{t}(x, t)=0$ on $\partial \Omega$, integration by parts applies to the first integral on the right and gives

$$
\int_{\Omega}\left\langle u_{t t}, u_{t}\right\rangle d x=-\int_{\Omega}\left\langle A(x) D u_{t}, D u_{t}\right\rangle d x+\int_{\Omega}\left\langle f_{u}(u) u_{t}, u_{t}\right\rangle d x .
$$

By the ellipticity condition, the above yields

$$
\frac{1}{2} \frac{d}{d t}\left(\int_{\Omega}\left|u_{t}\right|^{2} d x\right)+\lambda \int_{\Omega}\left|D u_{t}\right|^{2} d x \leq \int_{\Omega}\left|f_{u}(u)\right|\left|u_{t}\right|^{2} d x .
$$

Using Hölder's inequality and (3.3), we can estimate the integral on the right of (3.6) as follows:

$$
\begin{aligned}
\int_{\Omega}\left|f_{u}(u)\right|\left|u_{t}\right|^{2} d x & \leq\left(\int_{\Omega}\left|f_{u}(u)\right|^{2} d x\right)^{\frac{1}{2}}\left(\int_{\Omega}\left|u_{t}\right|^{4} d x\right)^{\frac{1}{2}} \\
& =\left(\Phi_{2}(t)\right)^{\frac{1}{2}}\left(\int_{\Omega}\left|u_{t}\right|^{4} d x\right)^{\frac{1}{2}} .
\end{aligned}
$$

Here, we denoted

$$
\Phi_{2}(t):=\int_{\Omega}\left|f_{u}(u(x, t))\right|^{2} d x \quad t \in\left(0, T_{0}\right) .
$$

Applying (3.2) with $\phi=u_{t}$ to the the last integral on the right of (3.7) we obtain

$$
\int_{\Omega}\left|f_{u}(u)\right|\left|u_{t}\right|^{2} d x \leq \Phi_{2}(t)^{\frac{1}{2}}\left(C \int_{\Omega}\left|D u_{t}\right|^{2} d x \int_{\Omega}\left|u_{t}\right|^{2} d x\right)^{\frac{1}{2}} .
$$

By Cauchy's inequality,

$$
\int_{\Omega}\left|f_{u}(u)\right|\left|u_{t}\right|^{2} d x \leq \frac{\lambda}{2} \int_{\Omega}\left|D u_{t}\right|^{2} d x+\frac{C \Phi_{2}(t)}{2 \lambda} \int_{\Omega}\left|u_{t}\right|^{2} d x .
$$

Substituting this inequality into (3.6) and rearranging, we have

$$
\frac{1}{2} \frac{d}{d t}\left(\int_{\Omega}\left|u_{t}\right|^{2} d x\right)+\frac{\lambda}{2} \int_{\Omega}\left|D u_{t}\right|^{2} d x \leq \frac{C \Phi_{2}(t)}{2 \lambda} \int_{\Omega}\left|u_{t}\right|^{2} d x .
$$


For $y(t)=\int_{\Omega}\left|u_{t}(x, t)\right|^{2} d x$, the above then implies for all $t \in\left(0, T_{0}\right)$,

$$
y^{\prime}(t) \leq \Phi_{3}(t) y(t), \quad \Phi_{3}(t):=\frac{C \Phi_{2}(t)}{\lambda} .
$$

This is a Gronwall's inequality, so that

$$
y(t) \leq y\left(t_{0}\right) e^{\Phi_{4}(t)} \quad \forall t_{0}>0, t \in\left(t_{0}, T_{0}\right) \quad \text { and } \quad \Phi_{4}(t):=\int_{t_{0}}^{t} \Phi_{3}(s) d s .
$$

Therefore,

$$
\int_{\Omega}\left|u_{t}(x, t)\right|^{2} d x \leq e^{\Phi_{4}(t)} \int_{\Omega}\left|u_{t}\left(x, t_{0}\right)\right|^{2} d x \quad \forall t_{0}>0, t \in\left(t_{0}, T_{0}\right) .
$$

By our assumption (3.3) and the definition of the functions $\Phi_{i}$ 's, we easily see that

$$
\Phi_{4}(t) \leq \frac{C}{\lambda} \int_{0}^{t} \int_{\Omega}\left|f_{u}\right|^{2} d x d s \leq \frac{C}{\lambda} \Phi_{1}(t) .
$$

Thus, we can take $\bar{\Phi}(t)$ in (3.4) to be $e^{\frac{C}{\lambda} \Phi_{1}(t)}$ to complete the proof of the lemma.

We now estimate the integral of $D u$ to provide the main part of the proof of our theorems.

Lemma 3.2. Suppose that there is a constant $C$ such that

$$
\left|f_{u}(u)\right| \leq C(|u|+1)
$$

and that

$$
\sup _{t \in(0, T)} \int_{0}^{t} \int_{\Omega}\langle f(u), u\rangle d x d t \leq \Phi_{*}(T) \quad \forall T \in\left(0, T_{0}\right)
$$

for some continuous function $\Phi_{*}$ on $(0, \infty)$.

Then there is a continuous function $\Phi^{*}$ on $(0, \infty)$ depending on $\Phi_{*}$ such that

$$
\sup _{t \in[0, T]} \int_{\Omega}|D u(x, t)|^{2} d x \leq \Phi^{*}(T)\left[\int_{\Omega}\left[\left|u_{t}\left(x, t_{0}\right)\right|^{2}+f\left(u\left(x, t_{0}\right)\right) u\left(x, t_{0}\right)\right] d x+1\right]
$$

for all $t_{0} \in\left(0, T_{0}\right)$ and $T \in\left(t_{0}, T_{0}\right)$.

Proof. First of all, testing the system for $u$ with $u$ and using (3.13), we easily get

$$
\int_{\Omega}|u|^{2} d x+2 \lambda \int_{0}^{t} \int_{\Omega}|D u|^{2} d x d t \leq \int_{\Omega}|u(x, 0)|^{2} d x+2 \int_{0}^{t} \int_{\Omega}\langle f(u), u\rangle d x d t .
$$

Therefore, for $\Phi_{5}(t)=2 \int_{\Omega}|u(x, 0)|^{2} d x+3 \Phi_{*}(t)$ the above and (3.13) imply

$$
\sup _{t \in(0, T)} \int_{\Omega}|u|^{2} d x+\lambda \int_{0}^{T} \int_{\Omega}|D u|^{2} d x d t \leq \Phi_{5}(T) .
$$

On the other hand, by (3.2), we have

$$
\int_{\Omega}|u|^{4} d x \leq C \int_{\Omega}|u|^{2} d x \int_{\Omega}|D u|^{2} d x
$$

so that

$$
\int_{0}^{T} \int_{\Omega}|u|^{4} d x d t \leq C \sup _{t \in(0, T)} \int_{\Omega}|u|^{2} d x \int_{0}^{T} \int_{\Omega}|D u|^{2} d x d t .
$$


Applying (3.15) to each factor on the right hand side we get

$$
\int_{0}^{T} \int_{\Omega}|u|^{4} d x d t \leq \frac{C}{\lambda} \Phi_{5}^{2}(T) .
$$

We now let

$$
F(t):=\int_{\Omega}\langle f(u(x, t)), u(x, t)\rangle d x, \quad t \in\left(0, T_{0}\right) .
$$

For any $t_{0} \in\left(0, T_{0}\right)$ we have

$$
F(t)=F\left(t_{0}\right)+\int_{t_{0}}^{t} \int_{\Omega} \frac{\partial}{\partial s}\langle f(u(x, s)), u(x, s)\rangle d x d s \quad \forall t \in\left(t_{0}, T_{0}\right) .
$$

Now, $\frac{\partial}{\partial s}\langle f(u(x, s)), u(x, s)\rangle \leq\left|f_{u}\right|\left|u_{s}\right||u|+|f(u)|\left|u_{s}\right|$ and the growth condition (2.1) on $f_{u}$ gives some constant $C$ such that $|f(u)| \leq C\left(|u|^{2}+1\right)$. Therefore, Young's inequality applies here to give $\left|f_{u}\right|\left|u_{s}\right||u|+|f(u)|\left|u_{s}\right| \leq C\left(\left|u_{s}\right|^{2}+|u|^{4}+1\right)$. Hence,

$$
F(t) \leq F\left(t_{0}\right)+C \int_{t_{0}}^{T} \int_{\Omega}\left(\left|u_{s}\right|^{2}+|u|^{4}+1\right) d x d s \quad t_{0}<t \leq T<T_{0} .
$$

On the other hand, by (2.1) and (3.15), we have

$\int_{0}^{t} \int_{\Omega}\left|f_{u}(u(x, s))\right|^{2} d x d s \leq C \int_{0}^{t} \int_{\Omega}\left(|u|^{2}+1\right) d x d s \leq C t\left(\Phi_{5}(t)+|\Omega|\right) \quad \forall t \in\left(0, T_{0}\right)$.

Thus, the condition (3.3) of the previous lemma holds here and we have the estimate (3.4) for the integral of $\left|u_{t}\right|^{2}$ over $\Omega$. This estimate and (3.16) are then used in (3.18) to provide a continuous function $\Phi_{6}(t)$ on $(0, \infty)$ such that

$$
\sup _{t \in\left(t_{0}, T\right]} F(t) \leq F\left(t_{0}\right)+\Phi_{6}(T)\left[\int_{\Omega}\left|u_{t}\left(x, t_{0}\right)\right|^{2} d x+1\right] \quad \forall T \in\left(t_{0}, T_{0}\right) .
$$

Finally, rewriting the system for $u$ as $-\operatorname{div}(A(x) D u)=f(u)-u_{t}$ and testing it with $u$ and using Young's inequality, we obtain

$$
\lambda \int_{\Omega}|D u|^{2} d x \leq \int_{\Omega}\langle f(u), u\rangle d x+\int_{\Omega}\left|u_{t}\right||u| d x \leq F(t)+\frac{1}{2} \int_{\Omega}\left(\left|u_{t}\right|^{2}+|u|^{2}\right) d x .
$$

Combining the above with (3.4), (3.15) and (3.19) we prove the lemma.

We are now ready to give the proof of our theorems.

Proof of Theorem 2.1. It is clear that the assumption (2.2) of the theorem allows us to apply Lemma 3.2 here so that if $u$ exists on $\left(0, T_{0}\right)$, then for any $t_{0} \in\left(0, T_{0}\right)$ we can find a continuous function $H_{t_{0}}(t)$ on $\left(t_{0}, \infty\right)$ such that

$$
\int_{\Omega}|D u(x, t)|^{2} d x \leq H_{t_{0}}(t) \quad \forall t \in\left(t_{0}, T_{0}\right) .
$$

This implies that $u(x, t)$ is BMO in $x$ so that (see [5] $) u(\cdot, t) \in L^{q}\left(\Omega, \mathbb{R}^{m}\right)$ for all $q>1$ if $t>t_{0}$. Since $u$ is smooth in $\Omega \times\left(0, t_{0}\right], u(\cdot, t)$ is also in $L^{q}(\Omega)$ for $t \in\left(0, t_{0}\right]$ (e.g., see [5]). Therefore, there exists a continuous function $C_{f, q}(t)$ on $(0, \infty)$ such that

$$
\|f(u(\cdot, t))\|_{L^{q}(\Omega)} \leq C_{f, q}(t) \quad \forall t \in\left(0, T_{0}\right) .
$$

In order to obtain the global existence of $u$ we will follow the theory in [1] which establishes that it suffices to show that certain Hölder norm of $u$ does not blow up in finite time. The following argument was used in [7, 9] but we will present 
some details here for the convenience of the reader. Let us fix a $p>2$ and consider $X=L^{p}\left(\Omega, \mathbb{R}^{m}\right)$. Let $\mathcal{A}$ be the realization of the operator $\operatorname{div}(A(x) D v)$ acting on functions in $X$ with vanishing boundary traces. That is,

$$
\mathcal{A} v=\operatorname{div}(A(x) D v) \quad \text { with } \operatorname{dom}(\mathcal{A})=W^{2, p}\left(\Omega, \mathbb{R}^{m}\right) \cap W_{0}^{1, p}\left(\Omega, \mathbb{R}^{m}\right) .
$$

For initial data $u_{0} \in W_{0}^{1, p}\left(\Omega, \mathbb{R}^{m}\right)$ we can abstractly write (1.1) as

$$
u_{t}=\mathcal{A} u+f(u), \quad u(0)=u_{0} .
$$

Under the smoothness assumptions $\mathbf{A})$ of $A(x)$ we easily see that $\mathcal{A}$ satisfies all the conditions in [4] to ensure the existence of the evolution operators

$$
\mathcal{U}(t, s) \in \mathcal{L}(X) \quad 0 \leq s \leq t<\infty
$$

such that

$$
u(t)=\mathcal{U}(t, 0) u_{0}+\int_{0}^{t} \mathcal{U}(t, s) f(u(s)) d s .
$$

We have the following estimate concerning the operator $\mathcal{U}(t, s)$. There exist positive numbers $\omega, C_{\gamma}$ such that for any $\gamma \in[0,1]$ and $0 \leq s \leq t<\infty$ (see (16.38) of [4]),

$$
\left\|\mathcal{A}^{\gamma} \mathcal{U}(t, s)\right\|_{\mathcal{L}(X)} \leq \frac{C_{\gamma} e^{-\omega(t-s)}}{(t-s)^{\gamma}}
$$

We now apply $\mathcal{A}^{\gamma}$ to (3.21) to have

$$
\mathcal{A}^{\gamma} u(t)=\mathcal{A}^{\gamma} \mathcal{U}(t, 0) u_{0}+\int_{0}^{t} \mathcal{A}^{\gamma} \mathcal{U}(t, s) f(u(s)) d s .
$$

Therefore, using (3.22) and (3.20) with $q=p$,

$$
\begin{aligned}
\left\|\mathcal{A}^{\gamma} u(t)\right\|_{X} & \leq\left\|\mathcal{A}^{\gamma} \mathcal{U}(t, 0) u_{0}\right\|_{X}+\int_{0}^{t}\left\|\mathcal{A}^{\gamma} \mathcal{U}(t, s) f(u(s))\right\|_{X} d s \\
& \leq C_{\gamma} t^{-\gamma} e^{-\omega t}\left\|u_{0}\right\|_{X}+\int_{0}^{t} C_{\gamma}(t-s)^{-\gamma} e^{-\omega(t-s)}\|f(u(s))\|_{X} d s \\
& \leq C_{\gamma} t^{-\gamma} e^{-\omega t}\left\|u_{0}\right\|_{X}+\max _{0 \leq s \leq t} C_{f, p}(s) \int_{0}^{t} C_{\gamma}(t-s)^{-\gamma} e^{-\omega(t-s)} d s .
\end{aligned}
$$

It is clear that the last quantity is a continuous function of $t \in(0, \infty)$. We just showed that the norm $\left\|\mathcal{A}^{\gamma} u(t)\right\|_{X}$ does not blow up in finite time. It is well known that the space $Y_{\gamma}=\operatorname{dom}\left(A^{\gamma}\right)$, with the graph norm $\|v\|_{Y^{\gamma}}=\left\|A^{\gamma} v\right\|_{X}$ and $X=L^{p}\left(\Omega, \mathbb{R}^{m}\right)$, is continuously embedded in $C^{\alpha}\left(\Omega, \mathbb{R}^{m}\right)$ if $0 \leq \alpha<2 \gamma-2 / p$. By (3.20), for any given $\gamma \in(0,1]$ we can choose $p$ large enough so that $\alpha>0$. Thus, the $C^{\alpha}$ norm of $u$ does not blow up in finite time and the global existence of $u$ then follows. The proof is complete.

The proof of Corollary 2.2 then follows.

Proof of Corollary 2.2, We need only show that the assumption (2.3),

$$
\sup _{t \in(0, T)} \int_{\Omega}|u(x, t)|^{p} d x \leq \Phi(T) \quad \forall T \in\left(0, T_{0}\right), p>1,
$$

implies (2.2) of Theorem 2.1. Since $f(u)$ has quadratic growth in $u$, one has

$$
\int_{0}^{T} \int_{\Omega}\langle f(u), u\rangle d x d t \leq C \int_{0}^{T} \int_{\Omega}\left(|u|^{3}+1\right) d x d t .
$$


If $p \geq 4$, then (2.2) holds trivially. Thus we only consider $p \in(1,3)$.

Taking $n=2, q=3$ and $r=p$ in (3.1), we have $\alpha=1-\frac{p}{3}$ and

Therefore,

$$
\left(\int_{\Omega}|u|^{3} d x\right)^{\frac{1}{3}} \leq C\left(\int_{\Omega}|u|^{p} d x\right)^{\frac{1}{3}}\left(\int_{\Omega}|D u|^{2} d x\right)^{\frac{3-p}{6}} .
$$

$$
\int_{\Omega}|u|^{3} d x \leq C \int_{\Omega}|u|^{p} d x\left(\int_{\Omega}|D u|^{2} d x\right)^{\gamma}, \quad \gamma=\frac{3}{2}-\frac{p}{2} \in(0,1) .
$$

Using the above in (3.24), we get

$$
\int_{0}^{T} \int_{\Omega}\langle f(u), u\rangle d x d t \leq C \int_{0}^{T} \int_{\Omega}|u|^{p} d x\left(\int_{\Omega}|D u|^{2} d x\right)^{\gamma} d t+C|\Omega| T .
$$

By the assumption (3.23), the above implies a constant $C$ such that

$$
\int_{0}^{T} \int_{\Omega}\langle f(u), u\rangle d x d t \leq C \Phi(T)\left(\int_{0}^{T} \int_{\Omega}|D u|^{2} d x d t\right)^{\gamma}+C|\Omega| T .
$$

As before, we test the system for $u$ with $u$ to get

$$
\lambda \int_{0}^{T} \int_{\Omega}|D u|^{2} d x d t \leq \int_{0}^{T} \int_{\Omega}\langle f(u), u\rangle d x d t+\frac{1}{2} \int_{\Omega}|u(x, 0)|^{2} d x .
$$

This and (3.25) yield

$$
\lambda \int_{0}^{T} \int_{\Omega}|D u|^{2} d x d t \leq C \Phi(T)\left(\int_{0}^{T} \int_{\Omega}|D u|^{2} d x d t\right)^{\gamma}+C|\Omega| T+\frac{1}{2} \int_{\Omega}|u(x, 0)|^{2} d x .
$$

Because $p>1$ we have $\gamma \in(0,1)$. A simple use of Young's inequality applying to the first term on the right gives

$$
C \Phi(T)\left(\int_{0}^{T} \int_{\Omega}|D u|^{2} d x d t\right)^{\gamma} \leq \frac{\lambda}{2} \int_{0}^{T} \int_{\Omega}|D u|^{2} d x d t+C(\lambda) \Phi(T)^{\frac{1}{1-\gamma}} .
$$

Using the above in (3.27), we obtain

$$
\frac{\lambda}{2} \int_{0}^{T} \int_{\Omega}|D u|^{2} d x d t \leq C(\lambda) \Phi(T)^{\frac{1}{1-\gamma}}+C|\Omega| T+\frac{1}{2} \int_{\Omega}|u(x, 0)|^{2} d x .
$$

Finally, a combination of (3.25) and (3.28) immediately gives a bound for the integral of $f(u) u$ and the condition (2.2) is verified. Our proof is then complete.

We end this section by giving the proof of Theorem 2.3 .

Proof of Theorem 2.3. Since $f(u)$ is of quadratic growth the assumption F1) is verified. We need only to check the condition (2.2) of Theorem 2.1 here. We see that

$$
\langle f(u), u\rangle=\sum_{i=1}^{m} b_{i} u_{i}^{2}-\sum_{i, j=1}^{m} c_{i j} u_{i}^{2}\left|u_{j}\right| \leq \sum_{i=1}^{m} b_{i} u_{i}^{2} .
$$

Hence, by testing the system of $u$ by $u$ and using the ellipticity condition $\mathbf{A}$ ), we easily obtain

$$
\frac{d}{d t} \int_{\Omega}|u|^{2} d x \leq 2 \int_{0}^{T} \int_{\Omega}\langle f(u), u\rangle d x d t \leq 2 \max _{i} b_{i} \int_{0}^{T} \int_{\Omega}|u|^{2} d x d t .
$$


We then have the following Gronwall's inequality so that

$$
\int_{\Omega}|u(x, t)|^{2} d x \leq e^{2 \max _{i} b_{i} t} \int_{\Omega}|u(x, 0)|^{2} d x .
$$

Therefore,

$$
\begin{aligned}
\int_{0}^{T} \int_{\Omega}\langle f(u), u\rangle d x d t & \leq \max _{i} b_{i} \int_{0}^{T} \int_{\Omega}|u|^{2} d x d t \\
& \leq \max _{i} b_{i} T e^{2 \max _{i} b_{i} T} \int_{\Omega}|u(x, 0)|^{2} d x .
\end{aligned}
$$

Thus, (2.2) of Theorem 2.1 is established and our theorem then follows.

\section{Sign CHANGing SOLUtions}

We conclude this paper by proving Theorem 2.4, which presents an example of a system discussed in Theorem 2.3 with sign changing solutions.

Now, for any $\varepsilon>0$ we consider the following system of two equations:

$$
\left[\begin{array}{l}
\left(u_{1}\right)_{t} \\
\left(u_{2}\right)_{t}
\end{array}\right]=\operatorname{div}\left(A\left[\begin{array}{c}
D u_{1} \\
D u_{2}
\end{array}\right]\right)+\left[\begin{array}{c}
u_{1}\left(2-\varepsilon\left|u_{1}\right|-\varepsilon\left|u_{2}\right|\right) \\
u_{2}\left(2-\varepsilon\left|u_{1}\right|-\varepsilon\left|u_{2}\right|\right)
\end{array}\right], \quad \text { in } \Omega \times(0, \infty)
$$

with $\Omega=(0, \pi) \times(0, \pi)$ and

$$
A=\left[\begin{array}{cc}
1 & -\frac{1}{2} \\
\frac{1}{2} & 1
\end{array}\right] .
$$

The matrix $A=\left(a_{i j}\right)$ satisfies the condition $\left.\mathbf{A}\right)$ because for $\eta=\left(\eta_{1}, \eta_{2}\right)$,

$$
\langle A \eta, \eta\rangle=\eta_{1}^{2}-\frac{1}{2} \eta_{1} \eta_{2}+\frac{1}{2} \eta_{2} \eta_{1}+\eta_{2}^{2}=|\eta|^{2} .
$$

The following functions are solutions to the problem (4.1) when $\varepsilon=0$ :

$$
v\left(x_{1}, x_{2}, t\right)=\left[\begin{array}{l}
v_{1}\left(x_{1}, x_{2}, t\right) \\
v_{2}\left(x_{1}, x_{2}, t\right)
\end{array}\right]=\left[\begin{array}{l}
\sin \left(t+\frac{\pi}{4}\right) \sin \left(x_{1}\right) \sin \left(x_{2}\right) \\
\cos \left(t+\frac{\pi}{4}\right) \sin \left(x_{1}\right) \sin \left(x_{2}\right)
\end{array}\right] .
$$

Indeed, we can check that $\left(v_{1}\right)_{t}=v_{2}, \Delta v_{1}=-2 v_{1},\left(v_{2}\right)_{t}=-v_{1}$ and $\Delta v_{2}=-2 v_{2}$ so that $v=\left[v_{1}, v_{2}\right]^{T}$ solves (4.1) when $\varepsilon=0$. Moreover, $v_{1}, v_{2}$ vanish on $\partial \Omega \times(0, \infty)$ and take on positive initial data when $t=0$. Nevertheless, $v_{1}, v_{2}$ are negative when $t=\pi$ !

The following claim gives the proof of Theorem 2.4

Claim. For $\varepsilon>0$ the system (4.1) has a unique classical solution $u=\left[u_{1}, u_{2}\right]^{T}$ that exists globally, vanishes on the lateral boundary $\partial \Omega \times(0, \infty)$ and takes positive initial data

$$
u_{1}\left(x_{1}, x_{2}, 0\right)=\frac{\sqrt{2}}{2} \sin \left(x_{1}\right) \sin \left(x_{2}\right), u_{2}\left(x_{1}, x_{2}, 0\right)=\frac{\sqrt{2}}{2} \sin \left(x_{1}\right) \sin \left(x_{2}\right) .
$$

Moreover, if $\varepsilon$ is sufficiently small, then this solution cannot stay nonnegative for all $t$.

Proof. System (4.1) can be rewritten as

$$
\begin{cases}u_{t}=\operatorname{div}(A D u)+2 u-\varepsilon g(u) & \text { in } \Omega \times(0, \infty), \\ u=0 & \text { on } \partial \Omega \times(0, \infty), \\ u(x, 0)=v(x, 0) & x \in \Omega .\end{cases}
$$


Here,

$$
g(u)=\left[\begin{array}{l}
u_{1}\left(\left|u_{1}\right|+\left|u_{2}\right|\right) \\
u_{2}\left(\left|u_{1}\right|+\left|u_{2}\right|\right)
\end{array}\right] \text { and } v(x, 0)=\frac{\sqrt{2}}{2}\left[\begin{array}{c}
\sin \left(x_{1}\right) \sin \left(x_{2}\right) \\
\sin \left(x_{1}\right) \sin \left(x_{2}\right)
\end{array}\right] .
$$

Because $f(u)=2 u-\epsilon g(u)$ is of quadratic growth, by Theorem 2.3 the solution $u$ is classical and exists globally. That is, $u$ exists on $(0, T]$ for every positive number $T$. Moreover, the last estimate in the proof of Theorem 2.3 with $\max _{i} b_{i}=2$ gives

$$
\sup _{0<t \leq T} \int_{0}^{t} \int_{\Omega}\langle f(u), u\rangle d x d t \leq 2 T e^{4 T} \int_{\Omega}|v(x, 0)|^{2} d x .
$$

The assumptions in Lemma (3.2) are satisfied with

$$
\Phi_{*}(T)=2 T e^{4 T} \int_{\Omega}|v(x, 0)|^{2} d x .
$$

Therefore, $u$ also has properties as in the proof of this lemma. By (3.16), we have

$$
\int_{0}^{T} \int_{\Omega}|u|^{4} d x d t \leq \frac{C}{\lambda}\left(2 \int_{\Omega}|u(x, 0)|^{2} d x+3 \Phi_{*}(T)\right)^{2}, \text { with } \lambda=1 .
$$

We now show that $u$ cannot stay nonnegative for all time. Let $k$ be a constant. We now write $w=(u-v) e^{-k t}$ and subtract the systems for $u, v$ and multiply the result with $e^{-k t}$ to have

$$
(w)_{t}=\operatorname{div}(A D w)+(2-k) w-\varepsilon e^{-k t} g(u) .
$$

Testing the above with $w$ and choosing $k>2$, we easily get for all $T>0$

$$
\sup _{t \in(0, T)} \int_{\Omega}|w|^{2} d x \leq \varepsilon \int_{0}^{T} \int_{\Omega} e^{-2 k t}|\langle g(u),(u-v)\rangle| d x d t .
$$

Here, by Young's inequality, there is a constant $K$ such that

$$
\int_{0}^{T} \int_{\Omega}|\langle g(u),(u-v)\rangle| d x d t \leq K\left(T \int_{\Omega}|v|^{2} d x+1+\int_{0}^{T} \int_{\Omega}|u|^{4} d x d t\right) .
$$

Therefore,

$$
\sup _{t \in(0, T)} \int_{\Omega}|w|^{2} d x \leq \epsilon K\left(T \int_{\Omega}|v|^{2} d x+1+\int_{0}^{T} \int_{\Omega}|u|^{4} d x d t\right) .
$$

Because the function $\Phi_{*}$ in (4.5) is independent of $\varepsilon$, the estimates (4.5) and (4.6) show that

$$
\lim _{\varepsilon \rightarrow 0} \sup _{t \in(0, T)} \int_{\Omega}|w|^{2} d x=0 \quad \forall T>0 .
$$

In particular, the above gives

$$
\lim _{\varepsilon \rightarrow 0} \int_{\Omega}|u(x, \pi)-v(x, \pi)|^{2} d x=0 .
$$

Because $v_{1}(x, \pi), v_{2}(x, \pi)<0$ in $\Omega$, the above shows that $u_{1}(x, \pi), u_{2}(x, \pi)$ cannot be nonnegative on $\Omega$ if $\varepsilon$ is sufficiently small. The proof is complete. 


\section{REFERENCES}

[1] Herbert Amann, Dynamic theory of quasilinear parabolic equations. II. Reaction-diffusion systems, Differential Integral Equations 3 (1990), no. 1, 13-75. MR1014726 (90i:35124)

[2] Herbert Amann, Dynamic theory of quasilinear parabolic systems. III. Global existence, Math. Z. 202 (1989), no. 2, 219-250, DOI 10.1007/BF01215256. MR1013086 (90i:35125)

[3] S. Ahmad, D. Le and V. Nguyen, Global and Blow Up Solutions to Cross Diffusion Systems on $3 D$ Domains, in preparation.

[4] Avner Friedman, Partial differential equations, Holt, Rinehart and Winston, Inc., New YorkMontreal, Que.-London, 1969. MR.0445088 (56 \#3433)

[5] Enrico Giusti, Direct methods in the calculus of variations, World Scientific Publishing Co., Inc., River Edge, NJ, 2003. MR,1962933 (2004g:49003)

[6] Konrad Horst Wilhelm Küfner, Invariant regions for quasilinear reaction-diffusion systems and applications to a two population model, NoDEA Nonlinear Differential Equations Appl. 3 (1996), no. 4, 421-444, DOI 10.1007/BF01193829. MR.1418589 (97m:35135)

[7] Le Dung, Global attractors and steady state solutions for a class of reaction-diffusion systems, J. Differential Equations 147 (1998), no. 1, 1-29, DOI 10.1006/jdeq.1998.3435. MR.1632736 (99f:35086)

[8] Dung Le, Cross diffusion systems on $n$ spatial dimensional domains, Indiana Univ. Math. J. 51 (2002), no. 3, 625-643, DOI 10.1512/iumj.2002.51.2198. MR1911048 (2003b:35090)

[9] Le Dung and Hal L. Smith, A parabolic system modeling microbial competition in an unmixed bio-reactor, J. Differential Equations 130 (1996), no. 1, 59-91, DOI 10.1006/jdeq.1996.0132. MR 1409023 (98c:92008)

[10] D. Le, L. Nguyen and T. Nguyen, Coexistence in Cross Diffusion systems, Indiana Univ. J. Math., Vol. 56, No. 4, pp.1749-1791, 2007.

[11] Yuan Lou and Wei-Ming Ni, Diffusion, self-diffusion and cross-diffusion, J. Differential Equations 131 (1996), no. 1, 79-131, DOI 10.1006/jdeq.1996.0157. MR1415047 (97i:35086)

[12] Nanako Shigesada, Kohkichi Kawasaki, and Ei Teramoto, Spatial segregation of interacting species, J. Theoret. Biol. 79 (1979), no. 1, 83-99, DOI 10.1016/0022-5193(79)90258-3. MR.540951 (80e:92038)

Department of Mathematics, University of Texas at San Antonio, One Utsa Circle, San Antonio, Texas 78249

E-mail address: dle@math.utsa.edu

Department of Mathematics, University of Texas at San Antonio, One UtSa Circle, San Antonio, Texas 78249 